\title{
Etude De L'Activité Antioxydante Des Huiles Essentielles De Plantes Aromatiques Et Médicinales Marocaines
}

\author{
Rachid Ismaili \\ Sara Houbairi \\ Laboratoire de Chimie appliquée et Environnement, \\ Faculté des Sciences et Techniques, Université Hassan1, \\ Settat, Maroc
}

\section{Lanouari Sanâa}

Laboratoire de Biologie, Santé et Environnement, Faculté des Sciences et Techniques, Université Hassan1, Settat, Maroc

\section{Moustaid Khadija \\ Lamiri Abdeslam}

Professeur d'Enseignement Supérieur, Faculté des Sciences et Techniques, Université Hassan1, Settat, Maroc

doi: 10.19044/esj.2017.v13n12p323 URL:http://dx.doi.org/10.19044/esj.2017.v13n12p323

\begin{abstract}
Morocco is a traditional supplier of medicinal and aromatic plants to the world. This activity has resulted to the exploitation of wild plants than dried plants for herbal needs and food flavors. Many species are used for the production of plant extracts and other aromatic essences mainly for the pharmaceutical and cosmetics industry. Among these metabolites, there are essential oils that are mixtures of aromatic substances present in the form of tiny droplets in the leaves, fruit peel, resin, branches, and wood. These oils, however, is endowed with antioxidant properties. This study aims to evaluate the antioxidant activity of essential oils of three moroccan aromatic and medicinal plants: Thymus vulgaris, Mentha spicata, and Citrus limonum. The oil extraction was performed by steam distillation in a Clevenger type apparatus. Also, the antioxidant activity of these oils was evaluated by the method of DPPH (2,2-diphenyl-1-picrylhydrazyl), in comparison with the antioxidant synthetic, ascorbic acid. In the present work, the results showed that the antioxidant activity of three essential oils studied by the trapping method of free radical is moderate. Also, the oil of Thymus vulgaris has a strong activity which exceeds that of ascorbic acid. This was followed by the
\end{abstract}


oil of Mentha spicata, while the lowest activity was observed in Citrus limonum.

Keywords: Essential oil, Thymus vulgaris, Mentha spicata, Citrus limonum, antioxidant activity.

\section{Résumé}

Le Maroc est un fournisseur traditionnel du marché mondial en plantes aromatiques et médicinales. Cette activité met en exploitation aussi bien des plantes spontanées que des plantes séchées pour les besoins d'herboristerie et les aromates alimentaires. Un grand nombre d'espèces sont utilisées pour la production des essences végétales et autres extraits aromatiques destinés essentiellement à l'industrie pharmaceutique et cosmétique. Parmi ces métabolites, on trouve les huiles essentielles qui sont des mélanges de substances aromatiques présentes sous forme de minuscules gouttelettes dans les feuilles, les écorces des fruits, la résine, les branches et le bois. Ces huiles représentent un groupe très intéressant, qui sont dotés de propriétés antioxydantes. Cette étude a pour objectif d'évaluer l'activité antioxydante des huiles essentielles de trois plantes aromatiques et médicinales marocaine: Thymus vulgaris, Mentha spicata et Citrus limonum. L'extraction des huiles a été réalisée par hydrodistillation dans un appareil de type Clevenger et l'activité antioxydante de ces huiles a été évaluée par la méthode de DPPH (2,2-diphenyl-1-picrylhydrazyl), en comparaison avec l'antioxydant synthétique, l'acide ascorbique. Dans le présent travail, les résultats obtenus ont montré que l'activité antioxydante des trois huiles essentielles étudiées selon la méthode de piégeage du radical libre est modérée et que l'huile du Thymus vulgaris a une forte activité qui dépasse celle de l'acide ascorbique suivie de l'huile du Mentha spicata tandis que la plus faible activité a été observée chez Citrus limonum.

Mots-clés : Huile essentielle, Thymus vulgaris, Mentha spicata, Citrus limonum, activité antioxydante.

\section{Introduction}

Les huiles essentielles (HE) représentent une source de molécules bioactives et font l'objet de nombreuses études pour leur éventuelle utilisation comme alternative pour la protection des aliments contre l'oxydation (Barkat \& Imène, 2011; Toure, 2015).

Le recours à ces huiles s'avère être un choix pertinent comme agents de conservation à la place des conservateurs synthétiques. Ceci nous a amené à étudier l'activité antioxydante des HE du T. vulgaris, M. spicata et du $C$. limonum. Le rôle de ces essences comme antioxydants naturels suscite de 
plus en plus d'intérêt pour la prévention et le traitement du cancer, des maladies inflammatoires et cardiovasculaires ; elles sont également utilisées comme additifs en industrie agroalimentaire, pharmaceutique et cosmétique (Houbairi et al., 2015). Cette étude s'intéresse à l'évaluation du pouvoir réducteur du radical libre DPPH (2,2- diphenyl-1- picrylhydrazyl) des HE issues du T. vulgaris, M. spicata et C. limonum.

\section{Matériel et Méthodes \\ Matériel végétal}

Les échantillons de T. vulgaris ont été cueillis dans la province de Tafilelt, $C$. limonum dans la province d'Agadir et $M$. spicata dans la province de Settat. Les cueillettes ont été réalisées pendant le mois d'Avril. Les feuilles du thym, de la menthe ainsi que les fruits du $C$. limonum ont été coupés à la main et mises dans des sacs cartonnés, transportés au laboratoire et conservé à température ambiante jusqu'à utilisation.

\section{Préparation des huiles essentielles}

Les HE ont été préparées par hydrodistillation, en utilisant un appareil de type Clevenger (Meyer-Warnod, 1984), dans un ballon de 5 litres contenant 2 litres d'eau distillée et surmonté d'une colonne de $60 \mathrm{~cm}$ de longueur et $2 \mathrm{~cm}$ de diamètre reliée à un réfrigérant.

Les extractions ont été répétées trois fois pour chaque $\mathrm{HE}$ afin de récupérer des volumes considérables. Après l'élimination des traces d'eau par du sulfate de sodium anhydre, les HE obtenues ont été stockées dans des petits flacons opaques et mises dans un réfrigérateur à $4^{\circ} \mathrm{C}$ jusqu'à leur utilisation pour les tests de l'activité antioxydante.

\section{Activité Antioxydante}

L'évaluation préliminaire de l'activité antioxydante des échantillons a été réalisée en utilisant la méthode du radical stable 2,2-diphenyl-1picrylhydrazyl (DPPH), qui a été initialement développée par Blois (1958). Le DPPH, de couleur violette en solution, présente un maximum d'absorption à $515 \mathrm{~nm}$. Le pouvoir antioxydant des HE testées a été estimé par comparaison avec un antioxydant naturel (Acide ascorbique). Tous les tests ont été réalisés avec trois répétitions pour chaque concentration.

L'activité du balayage du radical DPPH a été mesurée selon le protocole décrit par Lopes-Lutz et al. (2008), où $100 \mu \mathrm{l}$ de chacune des solutions méthanoïques des $\mathrm{HE}$ testées à différentes concentrations sont mélangées avec $1300 \mu \mathrm{l}$ d'une solution méthanoïque de DPPH (0,004\%). Après une période d'incubation de 30 minutes à la température de laboratoire, l'absorbance est lue à $517 \mathrm{~nm}$. L'inhibition du radical libre DPPH par l'acide ascorbique a été également analysée pour comparaison. 
La cinétique de la réaction et les paramètres de calcul de l'activité antioxydante pour l'acide ascorbique et les HE étudiées, ont été calculés.

Détermination du pourcentage d'inhibition (Khoudali et al., 2014)

L'inhibition des radicaux libres en pourcentages (I\%) est calculée en utilisant la formule suivante :

$$
\text { I \% }=[1-(\text { AbStest } / \text { Abscontrôle })] \times 100
$$

Abstest : absorbance de l'échantillon.

Abscontrôle : absorbance contrôle négatif.

Tous les essais ont été effectués en triple.

La cinétique des réactions des HE et de l'acide ascorbique avec le DPPH a été inscrite à chaque concentration examinée. Les concentrations en $\mathrm{HE}$ et en acide ascorbique, en fonction des pourcentages du DPPH inhibés, ont été tracées à la fin des réactions afin d'obtenir l'index $\mathrm{IC}_{50}$. Ce paramètre est défini comme la concentration d'antioxydant requise pour diminuer la concentration du DPPH initiale de 50\%.

\section{Détermination du temps d'équilibre TEC50 (Houbairi et al., 2015)}

Le paramètre $\mathrm{TEC}_{50}$ est défini comme le temps atteint à l'équilibre avec une concentration d'antioxydant égale à $\mathrm{IC}_{50}$. Ce temps est déterminé graphiquement.

\section{Détermination du paramètre d'efficacité anti-radicalaire EA (Houbairi et $a l ., 2015$ )}

Les deux facteurs $\mathrm{IC}_{50}$ et $\mathrm{TEC}_{50}$ peuvent être combinés afin d'obtenir le paramètre d'efficacité anti-radicalaire:

\section{Résultats et Discussion}

$$
\mathbf{E A}=\mathbf{T E C}_{50} / \mathbf{I C}_{50}
$$

\section{Courbe d'étalonnage de la solution DPPH}

Une évaluation de la stabilité et l'intervalle de linéarité des solutions de DPPH ont été évalués et les résultats sont présentés sur le graphique cidessous. Six solutions du DPPH $(0,5,10,15,30$ et $60 \mu \mathrm{M})$ à base du méthanol ont été testées. 


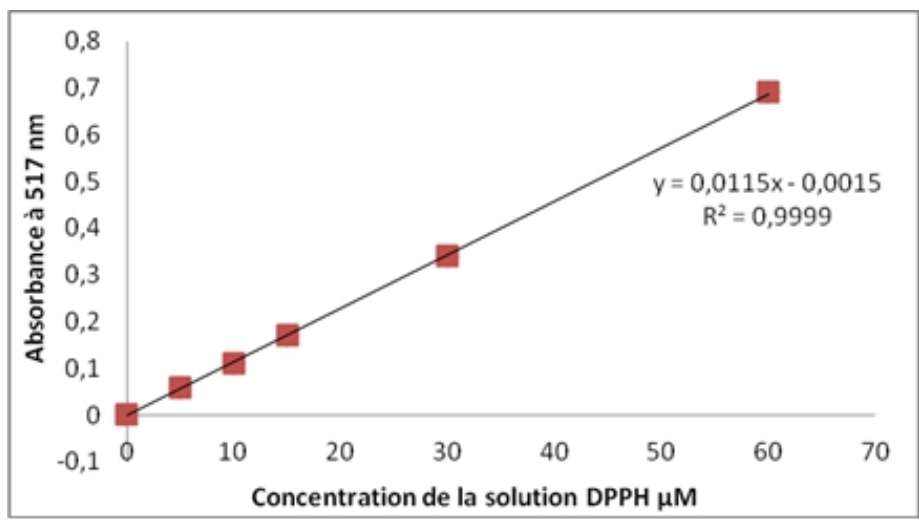

Figure 1. Courbe d'étalonnage de la solution de DPPH

Les absorbances à 0 et à 60 min ont été mesurés pour chaque concentration, on observe qu'il n'y a pas de différence significative dans l'absorbance entre 0 et $60 \mathrm{~min}$ pour les concentrations testées et une très bonne linéarité de l'absorbance en fonction de la concentration.

\section{Cinétique De La Réaction}

La cinétique de réduction du radical libre DPPH obtenue est indiquée pour chaque concentration de l'acide ascorbique sur la Figure 2, des HE de T. vulgaris (Figure 3), M. spicata (Figure 4), et C. limonum (Figure 5).

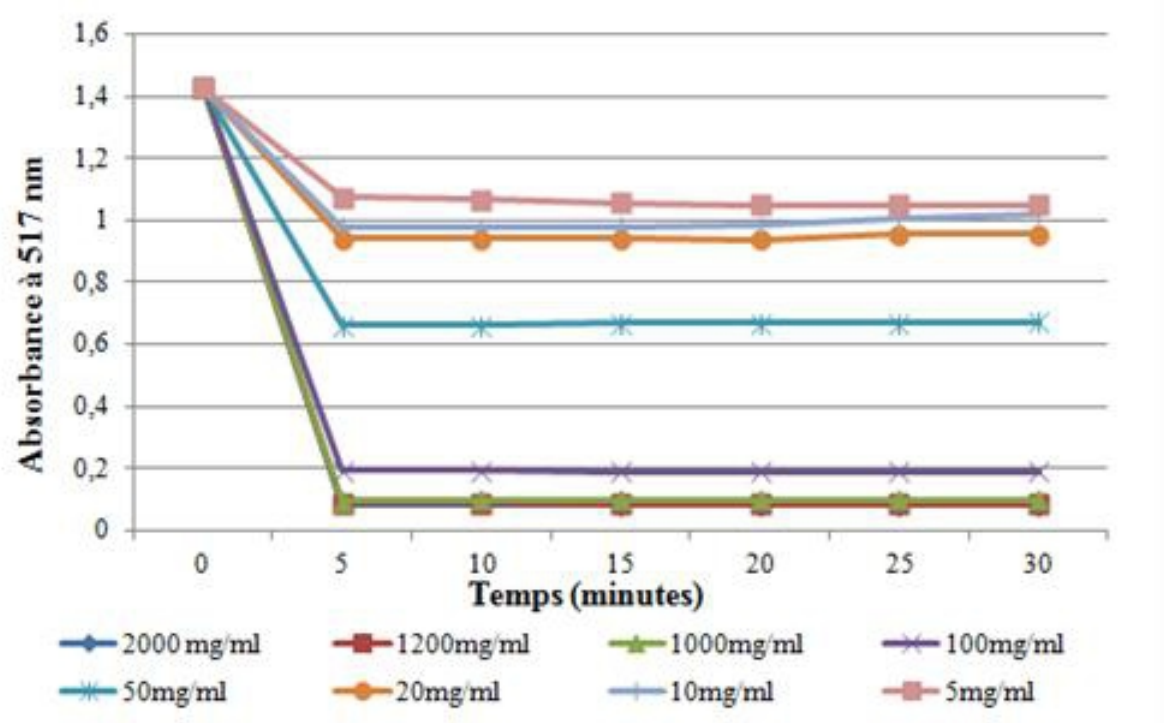

Figure 2 : Cinétique de réduction du DPPH obtenue avec l'acide ascorbique 


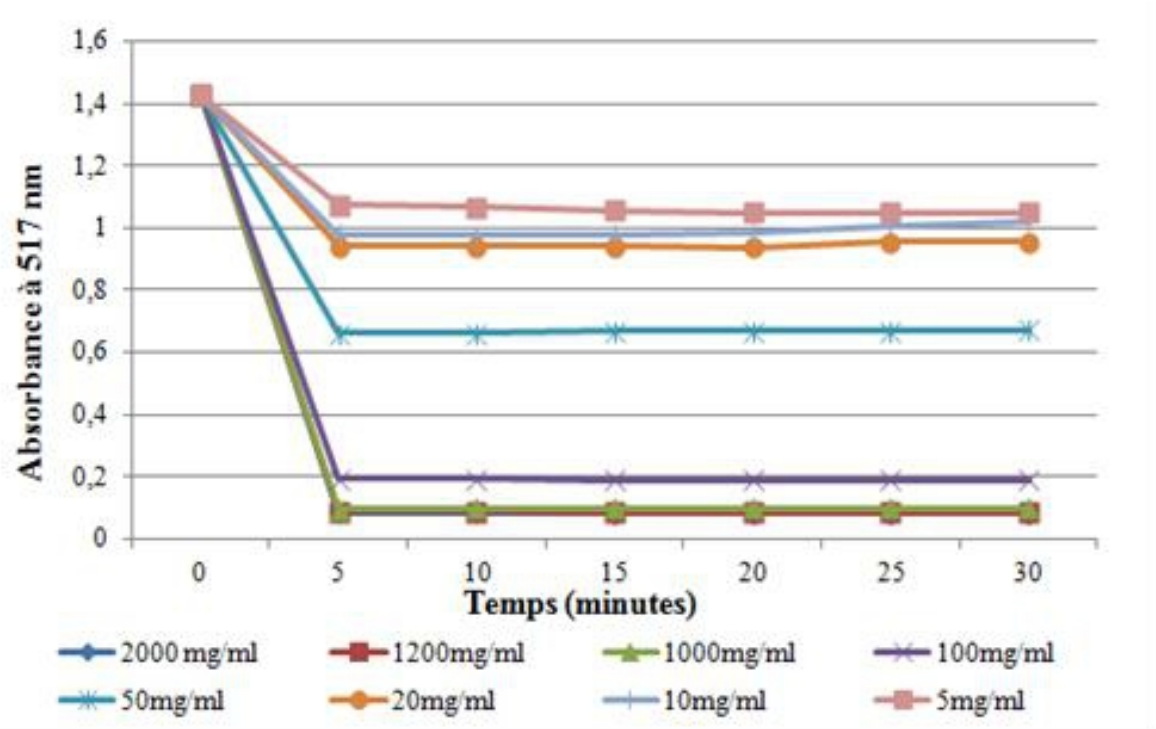

Figure 3. Cinétique de réduction du DPPH obtenue avec l'huile essentielle de T. vulgaris

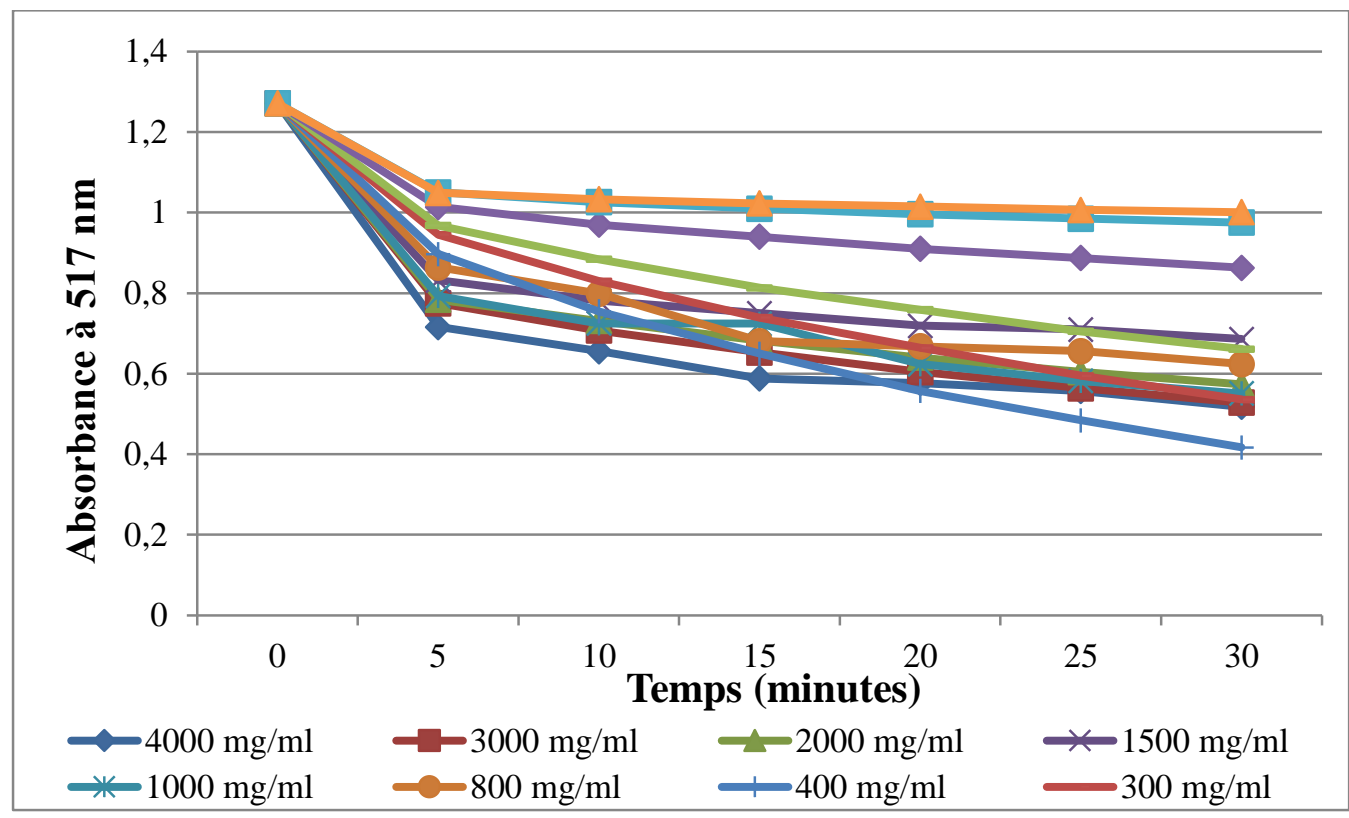

Figure 4 : Cinétique de réduction du DPPH obtenue avec l'huile essentielle du M. spicata. 


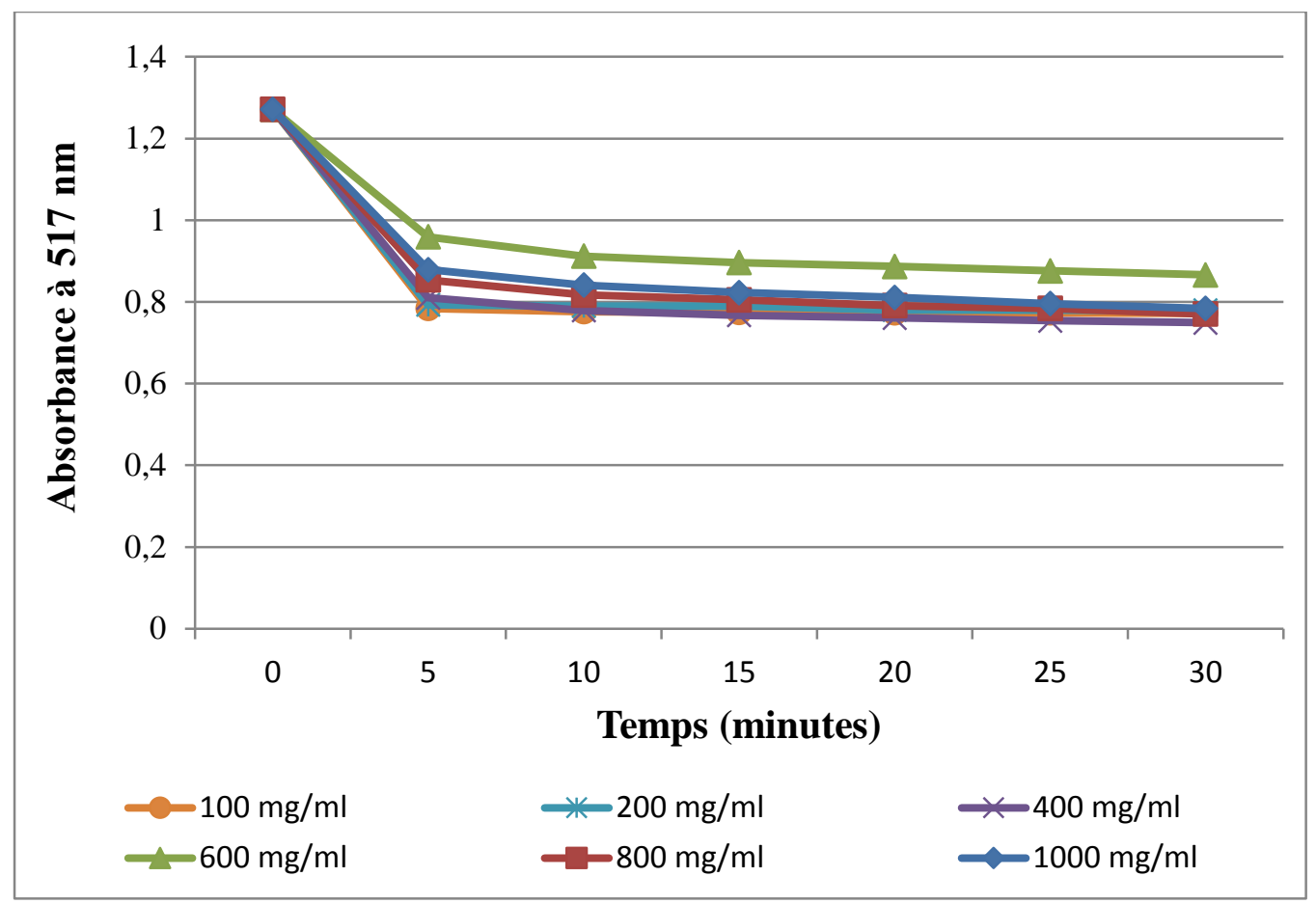

Figure 5. Cinétique de réduction du DPPH obtenue avec l'huile essentielle du C. limonum

Pour tous composés examinés (Acide ascorbique et HE), la réaction est biphasée, avec une baisse rapide dans l'absorbance dans les premières minutes, suivies d'une étape plus lente, jusqu'à ce que l'équilibre soit atteint, alors on distingue deux zones:

- Zone à forte cinétique de piégeage du radical observée au bout des cinq premières minutes pour l'acide ascorbique, l'HE de $M$. spicata, l'HE du C. limonum et l'HE du T. vulgaris.

- Zone à faible cinétique de piégeage du radical DPPH ou zone de tendance vers l'équilibre constatée après les 5 minutes pour l'acide ascorbique, l'HE du T. vulgaris, l'HE du M.spicata et l'HE du $C$. limonum.

Les résultats obtenus montrent que la réaction entre le DPPH et l'acide ascorbique, l'HE du C. limonum et l'HE du T. vulgaris, atteint un équilibre au bout d'un temps court par rapport à l'HE du M. spicata.

\section{Détermination Du Pourcentage D'inhibition}

La mesure de l'absorbance a été effectuée par spectrophotométrie à $517 \mathrm{~nm}$ et à partir des résultats obtenus, les pourcentages d'inhibition ont été calculés. Les valeurs obtenues ont permis de tracer les courbes représentant la variation du pourcentage d'inhibition en fonction des concentrations de 
l'acide ascorbique (Figure 6), de l'HE du M. spicata (Figure 7), l'HE du $T$. vulgaris (Figure 8), et l'HE du C. limonum (Figure 9).

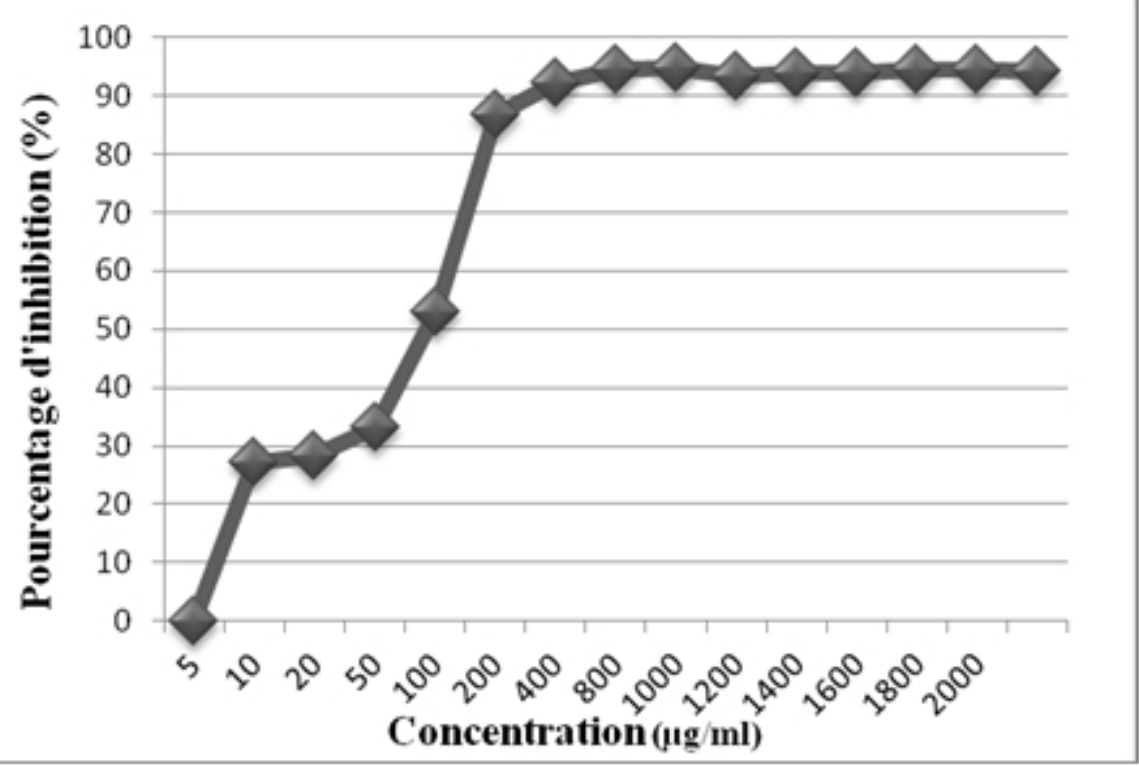

Figure 6. Pourcentage d'inhibition du DPPH en fonction des concentrations de l'acide ascorbique

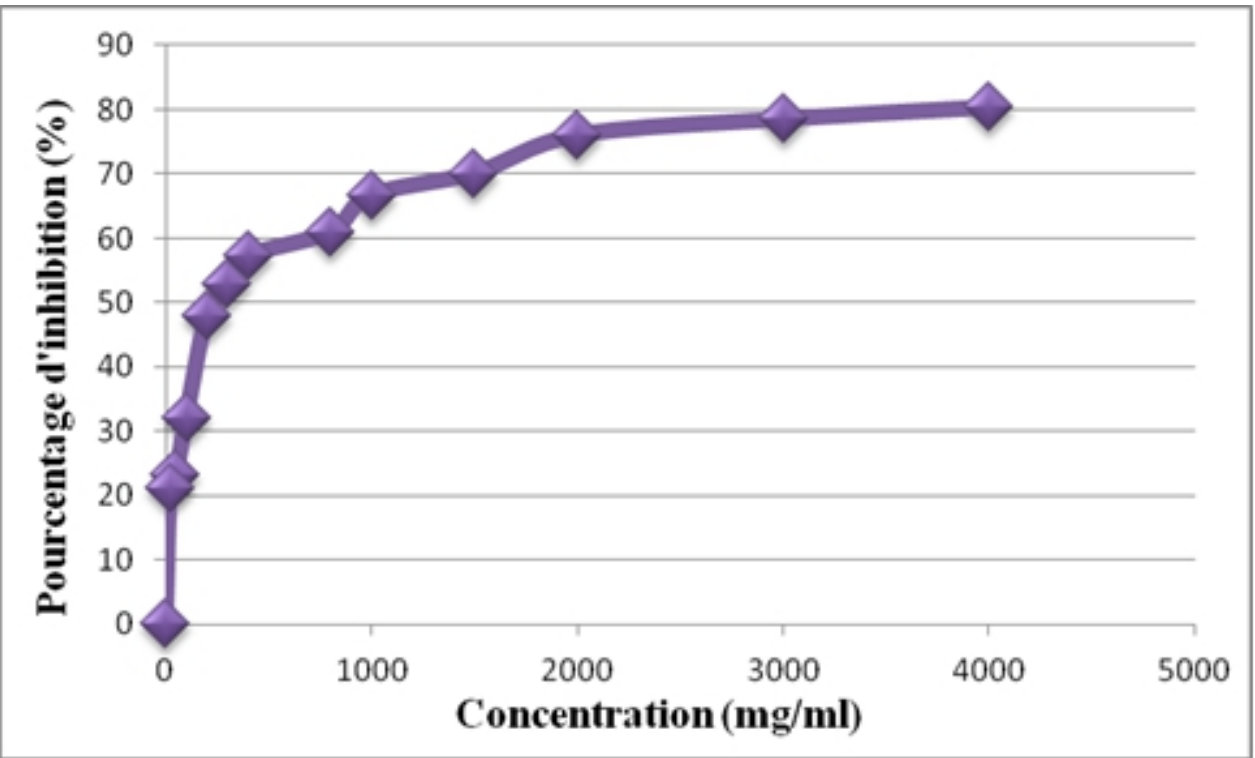

Figure 7. Pourcentage d'inhibition du DPPH en fonction des concentrations de l'HE $M$. Spicata 


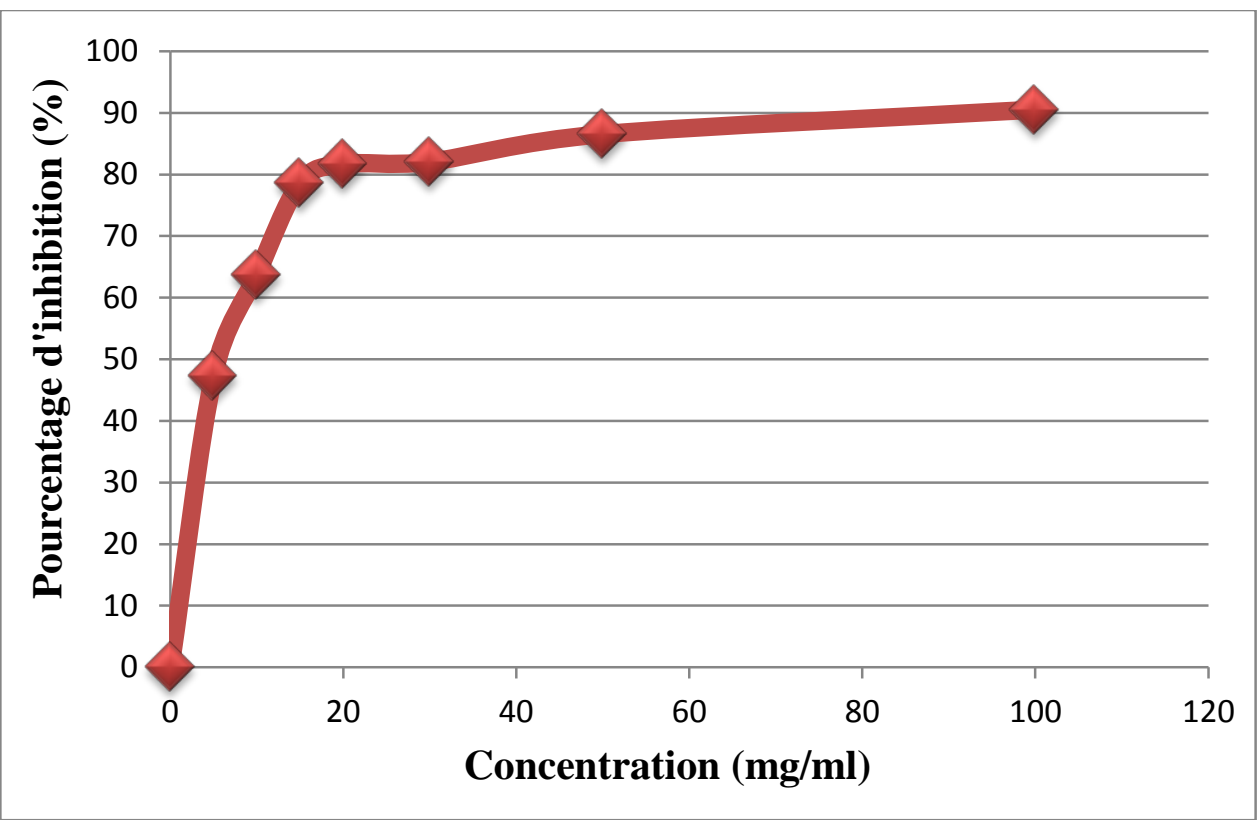

Figure 8. Pourcentage d'inhibition du DPPH en fonction des concentrations de l'HE du $T$. vulgaris

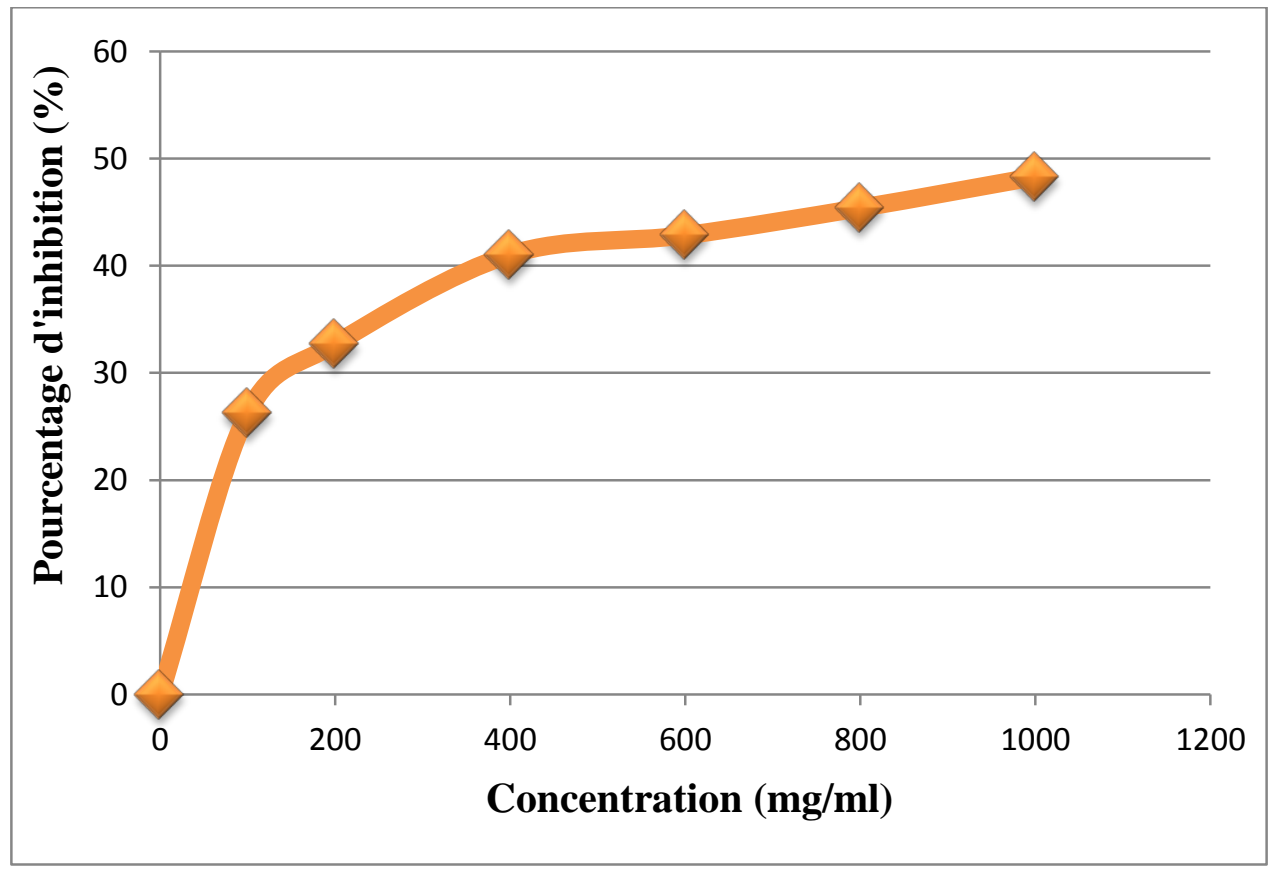

Figure 9. Pourcentage d'inhibition du DPPH en fonction des concentrations de l'HE du $C$. limonum 
Le pourcentage d'inhibition du radical libre augmente avec l'augmentation de la concentration soit pour l'acide ascorbique ou pour les HE testées.

Pour une concentration de $2000 \mathrm{mg} / \mathrm{ml}$, l'acide ascorbique a révélé un pourcentage d'inhibition du radical libre DPPH de $95 \%$, tandis que pour les concentrations d'HE du M. spicata $(4000 \mathrm{mg} / \mathrm{ml})$, du T. vulgaris (100 $\mathrm{mg} / \mathrm{ml})$ et du C. limonum $(1000 \mathrm{mg} / \mathrm{ml})$ les pourcentages d'inhibition obtenus sont respectivement $81,21 \pm 2,33 \%, 90 \pm 3,71 \%$ et $48,50 \pm 2,04 \%$.

\section{Détermination d'IC 50}

$\mathrm{IC}_{50}$ est inversement lié à la capacité antioxydante d'un composé, car il exprime la quantité d'antioxydant requise pour diminuer la concentration du radical libre de $50 \%$. Plus la valeur d' $\mathrm{IC}_{50}$ est basse, plus l'activité antioxydante d'un composé est grande. Les calculs des $\mathrm{IC}_{50}$ sont schématisés sur la Figure 10.

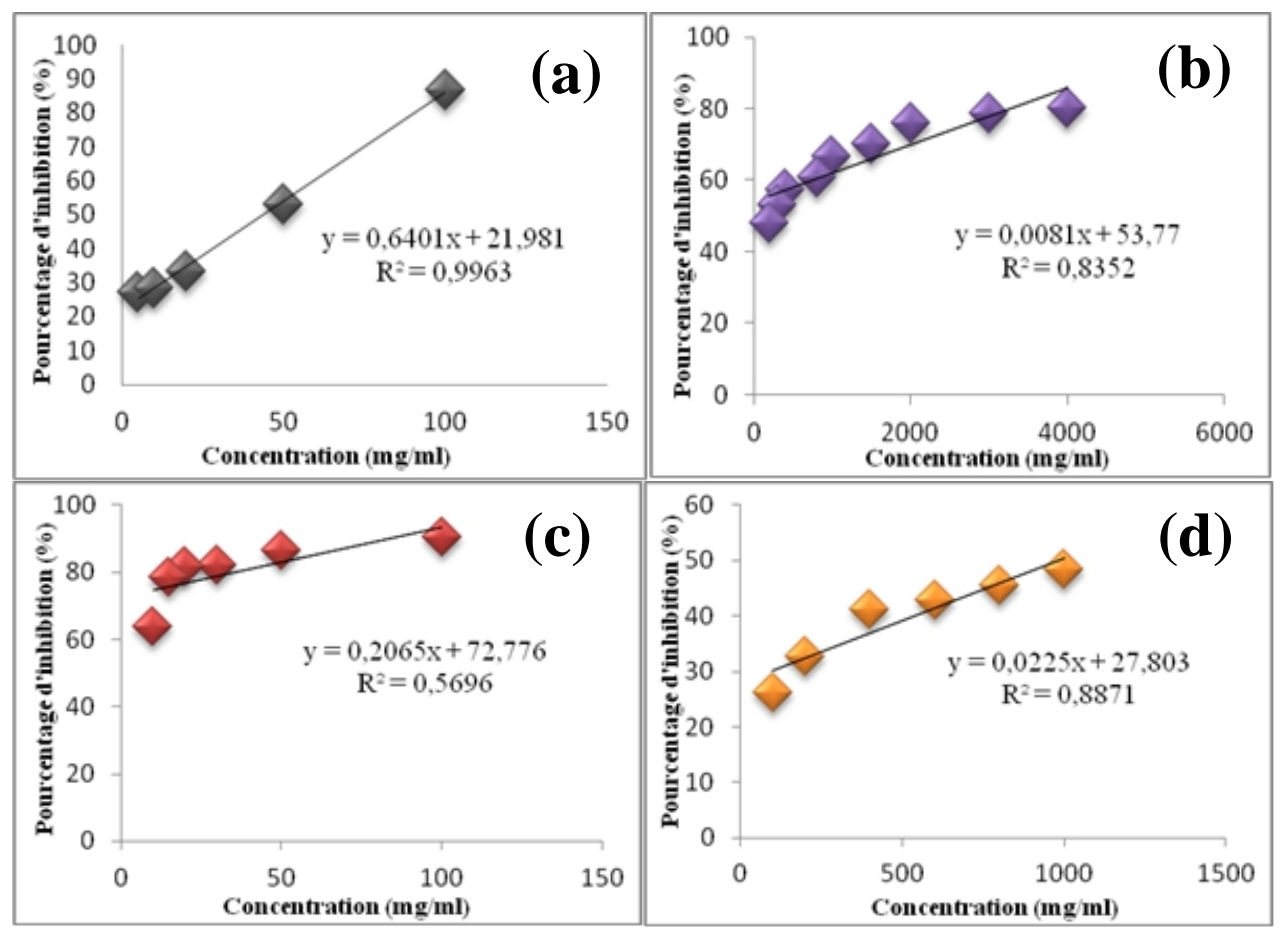

Figure 10. Calculs des $\mathrm{IC}_{50}$ pour : (a) : acide ascorbique ; (b) : HE du M. spicata ; (c) HE du T. vulgaris; (d) HE du C. limonum

Les valeurs d' $\mathrm{IC}_{50}$ déterminées graphiquement en $\mathrm{mg} / \mathrm{ml}$ exprimant la concentration efficace des différentes HE sont présentées dans le Tableau 1. 
Tableau 1. Résultat du test antioxydant exprimant la concentration efficace $50 \%$ en $\mathrm{mg} / \mathrm{ml}$.

\begin{tabular}{cc}
\hline HE/standard & IC $_{50} \pm$ Ecart type \\
\hline Acide ascorbique & $44,18 \pm 1,23$ \\
\hline Thymus vulgaris & $4,57 \pm 0,80$ \\
Mentha spicata & $201,34 \pm 2,58$ \\
Citrus limonum & $1000 \pm 3,20$ \\
\hline
\end{tabular}

L'HE du Citrus limonum ramène le radical libre stable 2,2 diphenyl-1picrylhydrazyl (DPPH) au diphenylpicrylhydrazine jaune-coloré avec un $\mathrm{IC}_{50}$ de $1000 \pm 3,20 \mathrm{mg} / \mathrm{ml}$ montrant une activité antioxydante inférieure à celle de l'acide ascorbique et des autres huiles testées. L'huile essentielle du Thymus vulgaris est l'antioxydant le plus efficace avec un $\mathrm{IC}_{50}$ de 4,57 \pm $0,80 \mathrm{mg} / \mathrm{ml}$.

\section{Détermination Du TEC 50}

L'état d'équilibre a été choisi comme période de mesure où il s'avère que la réaction progresse lentement. Le temps à l'état d'équilibre dépend de la réactivité des antioxydants et des concentrations utilisées.

L'acide ascorbique réagit d'une façon plus rapide avec le DPPH avec $\mathrm{TEC}_{50}=4,64 \pm 0,31$. Le temps d'équilibre des HE étudiées est de 23,50 \pm 1,11 pour T. vulgaris, $21.12 \pm 0.12$ pour $M$. spicata et $10,52 \pm 1,30$ pour $C$. limonum. Pour caractériser l'efficacité de ces antioxydants, on calcule le paramètre d'efficacité anti-radicalaire.

\section{Paramètre D'efficacité Anti-radicalaire}

L'efficacité anti-radicalaire, qui combine les deux paramètres $\left(\mathrm{IC}_{50}\right.$ et $\mathrm{TEC}_{50}$ ) a été définie afin de caractériser facilement le comportement d'une substance en tant qu'antioxydant. Les paramètres de calcul de l'activité antioxydante sont résumés dans le Tableau 2.

Tableau 2. Les paramètres de calcul de l'activité antioxydante

\begin{tabular}{cccc}
\hline & IC $\mathbf{5 0}(\mathbf{m g} / \mathbf{m l})$ & TEC $_{\mathbf{5 0}}(\mathbf{m n})$ & $\mathbf{E A}(\mathbf{m l} / \boldsymbol{\mu g} . \mathbf{m n})$ \\
\hline $\begin{array}{c}\text { Acide } \\
\text { ascorbique }\end{array}$ & $44,18 \pm 1,23$ & $4,64 \pm 0,31$ & $0,105 \pm 0,005$ \\
Thymus vulgaris & $4,57 \pm 0,80$ & $23,50 \pm 1,11$ & $5,142 \pm 0,210$ \\
Mentha spicata & $201,34 \pm 2,58$ & $21,12 \pm 0,12$ & $0,104 \pm 0,001$ \\
Citrus limonum & $1000 \pm 3,20$ & $10,52 \pm 1,30$ & $0,010 \pm 0,002$ \\
\hline
\end{tabular}

D'après ces résultats, l'HE du T. vulgaris possède l'activité antioxydante la plus élevée avec une efficacité anti-radicalaire de 5,142 \pm $0,210 \mathrm{ml} / \mu \mathrm{g} . \mathrm{mn}$ par rapport à l'acide ascorbique et aux HE testées. Par contre l'HE du C. limonum a présenté l'activité la plus faible avec une efficacité de $0,010 \pm 0,002 \mathrm{ml} / \mu \mathrm{g} . \mathrm{mn}$. 


\section{Conclusion}

L'étude de l'activité antioxydante des HE selon la méthode de piégeage du radical libre DPPH a montré que tous les huiles étudiées possèdent une activité antioxydante modérée et que l'HE du T. vulgaris a une forte activité qui dépasse celle de l'acide ascorbique.

Cette étude laisse entrevoir des perspectives d'application dans les domaines de l'industrie alimentaire, cosmétique et pharmaceutique. Les résultats obtenus pourraient contribuer à la valorisation de ces plantes aromatiques et médicinales marocaines.

\section{References :}

1. Barkat, M. \& Imène, L. (2011). Composition chimique et activité antioxydante de l'huile essentielle des fleurs sèches de Lavandula officinalis. Revue de génie industriel, $6:$ 46-54.

2. Blois, M.S. (1958). Antioxidant determinations by the use of a stable free radical. Nature, 181: 1199-1200.

3. De Pooter, H.L., Schamp, N. (1986). Comparison of the volatile composition of some Calamintha saturea species. In: Progress in essential oil research. Ed. Brunk EJ. Walter De Gruyter, Berlin: 139150.

4. Houbairi, S., Elmiziani, I., Lamiri, A., \& Essahli, M. (2015). Comparison of the Antioxidant Activity of Aromatic Medicinal Plants of Moroccan Origin. European Journal of Medicinal Plants, 10 (4): 1-10.

5. Khoudali, S., Benmessaoud, D., Essaqui, A., Zertoubi, M., \& Azzi, M. (2014). Etude de l'activité antioxydante et de l'action anti corrosion de l'extrait méthanolique des feuilles du palmier nain (Chamaerops humilis L.) du Maroc. J. Mater. Environ. Sci., 5(3): 887-898.

6. Lopez-tutz, D.S., Alviano, D.S., Alviano, C.P., \& Kolodziejczyk, P. (2008). Screening of chemical composition, antimicriobal and antioxidant activities of artemisia essential oils. Phytochemistry 69:1732-1738.

7. Meyer-Warnod, B. (1984). Natural essential oils: extraction processes and applications to some major oils, Perfumer \& Flavorist 9, 93-103.

8. Toure, D. (2015). Etudes chimique et biologique des huiles essentielles de quatre plantes aromatiques médicinales de cote d'ivoire. Thèse de Doctorat. Organic chemistry. Université Felix Houphoeut Boigny, Côte d'Ivoire, French. 